\title{
Cordomas del clivus. Consideraciones del tratamiento quirúrgico endoscópico
}

\section{Chordomas of the clivus. Concerns of endoscopic surgical treatment}

\section{Omar López Arbolay ${ }^{1}$ Marlon Ortiz Machín ${ }^{2}$, Alejandro Enrique Coronado Rosales ${ }^{3}$, Peggys O Cruz Pérez ${ }^{2}$, Pedro Pablo Gutiérrez Crespo ${ }^{3}$}

${ }^{1}$ Especialista de Segundo Grado en Neurocirugía, Profesor e Investigador Titular, Doctor en Ciencias Médicas, Doctor en Ciencias, Hospital Hermanos Ameijeiras. Cuba.

${ }^{2}$ Especialista de Segundo Grado en Neurocirugía, Profesor Asistente. Hospital Hermanos Ameijeiras.

${ }^{3}$ Especialista en I grado de Neurocirugía. Hospital Hermanos Ameijeiras.

\section{Resumen}

Introducción: Los cordomas del clivus son neoplasias de lento crecimiento, originadas de restos de la notocorda, que invaden el hueso y tienen alto índice de recurrencia. Objetivo: Caracterizar el comportamiento de esta entidad en nuestro centro en cuanto a los aspectos clínicos, radiológicos, terapéuticos y evolutivos. Método: Se realizó un estudio descriptivo retrospectivo en el período comprendido entre enero de 2010 a diciembre de 2015. La muestra fue de 10 pacientes, 6 masculinos y 4 femeninos, con una edad promedio de 55 años. Se realizó en todos los casos un abordaje endonasal endoscópico extendido al clivus, seguido de radioterapia de intensidad modulada (IMRT) hipofraccionada o 3D conformada Resultados: Las alteraciones clínicas más frecuentes fueron: la cefalea y la diplopía. Predominaron los cordomas clásicos al final de la sexta década de vida y en el sexo masculino. Se logró una resección completa de la lesión en el $80 \%$ de los casos y un control de la enfermedad a los 4 años en el $90 \%$. Existieron aceptables complicaciones y un fallecido que presentó fístula del líquido cefalorraquídeo y meningitis. Conclusiones: El abordaje endonasal endoscópico extendido al clivus constituye una opción quirúrgica excelente y prometedora, lográndose una resección quirúrgica radical de la lesión con aceptables complicaciones.

Palabras clave: Notocorda, cordoma del clivus, abordaje endoscópico.

\section{Abstract}

Introduction: Chordomas of clivus are slow growing neoplastic lesions originate by remnant of notochord and invade bone tissue with high rate of recurrence. Objective: To characterize the characteristics of this entity in our Hospital based on it's clinical, radiological, therapeutical and evolutionary aspects. Method: A retrospective descriptive study was performance from the period of January 2010 to December 2015. The sample was 10 patients, 6 men and 4 women, with an average age of 55 years. The surgical approach utilized on all patients was an endoscopic endonasal approach extended to the clivus, supported by intensity modulated radiotherapy (IMRT) hypofractioned or conformed 3D. Results: Headaches and diplopia was the most frequent clinical symptoms. Prevailed classic chordomas with a higher occurrence rate in males with aged 60 and above. Gross total resection was achieved in $80 \%$ and $90 \%$ of control rate during four year. Complications rates was little and one patient death with CSF fistula and meningitis. Conclusion: Extended endonasal endoscopic approach to the clivus is an excellent and promising surgical option with radical tumour resection and admissible complications.

Key words: Notochord, clivus chordoma, endoscopic approach.

\section{Correspondencia a:}

Dr. Omar López Arbolay

Jefe Neurocirugia HHA

neuroc@hha.sld.cu X 


\section{Introducción}

Los cordomas del clivus representan aproximadamente del 1 al $4 \%$ de los tumores malignos del hueso, con una incidencia de $0,8 \%$ por 100.000 habitantes, con predominio del sexo masculino entre 50 y 70 años de edad; se originan de las células remanentes indiferenciadas de la notocorda que residen en el esqueleto axial.

La presentación clínica depende de la localización y el grado de extensión, cursa mayormente con diplopía, seguida por parálisis del VI nervio craneal de forma típica pero también puede presentar parálisis del IX, X y XII nervios craneales, incluso una neuralgia trigeminal, cefalea, pérdida de la visión, trastornos endocrinos por invasión de la región selar, hemorragias intracraneales y epistaxis ${ }^{1,2}$.

Se presentan como una lesión ocupativa de la región clival bien circunscrita, con destrucción de esta, pobre captación de contraste iodado en la tomografía axial computarizada (TAC) y de gadolineo en la (RMN), con calcificaciones intratumorales presentes sobre todo en la variante condroide, aumento en la captación de la fluorodeoxyglucosa indicando hipermetabolismo, además de una extensión a nasofaringe, tallo encefálico, región selar y otros sitios ${ }^{3}$.

El diagnóstico definitivo es histopatológico; de este existen 3 variantes, la clásica, la condroide y la desdiferenciada. Inmunohistoquímicamente las células neoplásicas muestran reactividad para la proteína S-100 la cual muestra su origen ectodérmico, citoqueratinas las cuales permiten diferenciarlas de neoplasias de células claras como la metástasis de adenocarcinoma renal, antígeno epitelial de membrana, la cathepsina $\mathrm{K}$ y $\mathrm{E}$, el factor de transcripción nuclear (brachyury) y rara vez para el antígeno carcinoembrionario ${ }^{4}$. Las metástasis son infrecuentes en esta entidad, cuando aparecen se encuentran principalmente en el pulmón, hígado y hueso.

Una extensa variedad de abordajes quirúrgicos son utilizados, siendo el abordaje transcraneal el más común, pero el advenimiento y revolución de la cirugía endoscópica ha permitido una resección de la lesión lo más completa posible en una gran parte de los cordomas clivales, con una disminución en la morbimortalidad intra y postoperatoria asociado además a una tasa más baja de complicaciones y del tiempo quirúrgico; siempre teniendo en cuenta una serie de factores como el grado de infiltración dural, la extensión lateral de la lesión y el volumen tumoral que son retos a superar ${ }^{5}$.

La radioterapia protónica ha mostrado una tasa de resultados favorables por encima de otras modalidades de radioterapia como son la radioterapia convencional, la ion terapia, la radiocirugía; debido al pico Bragg que es el depósito de energía final, que permite habilitar y mantener una dosis efectiva de energía en el tejido y elimina la dosis de otros puntos $^{6}$. La quimioterapia no ha comprobado beneficios. La inmunoterapia muestra resultados alentadores como son la combinación de cetuximab con natural killer $(\mathrm{NK})^{7}$.

La supervivencia libre de enfermedad está en dependencia del grado de resección de la lesión, el tipo histológico, el tamaño de la lesión y el tipo de radioterapia empleada ${ }^{8,9}$. Las complicaciones quirúrgicas más comunes son la parálisis de nervios craneales, seguido de la fistula del líquido cefalorraquídeo, meningitis y hematomas.

A pesar de los avances alcanzados en los estudios neu- roimagenológicos y la perfección de los abordajes quirúrgicos, el índice de recidiva y morbilidad es elevado.

\section{Método}

Se realizó un estudio descriptivo retrospectivo en el período comprendido entre enero de 2010 a diciembre de 2015. La muestra fue de 10 pacientes, 6 masculinos y 4 femeninos, con una edad promedio de 55 años. En todos los casos se realizó una Resonancia Magnética Nuclear (RMN) de cráneo contrastada para identificar las características del tumor, así como medir el volumen tumoral según la fórmula del elipsoide en $\mathrm{cm}^{3}, V=4 / 3 \pi a b c$, donde $\mathrm{a}$, b y c son el largo, ancho y altura respectivamente.

Se realizó en todos los casos un Abordaje Endonasal Endoscópico Extendido al Clivus independientemente de su extensión en al plano coronal con la filosofía de la descompresión del tallo cerebral como objetivo cardinal de medial a lateral. Si el tumor tenía una extensión posterolateral a la región petroclival o al ángulo pontocerebeloso se extendió el abordaje al ápex petroso por vía transesfenoidal medial o transpterigoideo infrapetroso y si el tumor se extendía al seno cavernoso se realizó una extensión del mismo al seno cavernoso respectivamente (Figura 1). Todos los casos se intervinieron con neuronavegación, monitoreo neurofisiológico y no se utilizó el doppler transoperatorio por no poseerlo en nuestro centro, no se planificó colocar drenaje espinal continuo posoperatorio inmediato en ningún caso. En caso de existir restos mayores de $3 \mathrm{~cm}$ laterales o posterolaterales, se planificó realizar un Key Hole Subtemporal o Retrosigmoideo respectivamente a los 3 meses de la cirugía inicial como parte de un proceso de tratamiento. En todos los casos se realizó radioterapia de intensidad modulada (IMRT) hipofraccionada con 54 gy en 27 sesiones o 3D conformada (en nuestro país carecemos de radioterapia con bomba de protones), aunque el grado de resección macroscópica fuese total, por la frecuente presencia de células satélites aisladas en los puntos más intrincados de la anatomía de la base craneal. Los resultados se mostraron en tablas de contingencia con números y porcientos, se utilizó un análisis de componentes categóricos principales para determinar relación entre las distintas variables y el tiempo medio de seguimiento fue de 5,9 años, aunque se extendió por 10 años en algunos casos.

\section{Resultados}

En el período de 2010 al 2015 fueron intervenidos en nuestro servicio 10 pacientes con diagnóstico de cordomas del clivus. Las edades oscilaron entre 23 y 69 años (media 55 años) de ellos 6 hombres y 4 mujeres (Tabla 1).

Presentación clínica (Tabla 1): Los pacientes comenzaron con cefalea asociada a diplopía en el 100\% de los casos; la hipoacusia estuvo presente en los casos 3 y 5; predominó el cordoma clásico en el $70 \%$; con un volumen tumoral promedio de $59,5 \mathrm{ml}^{3}$. Se apreció en el $100 \%$ de los casos erosión del clivus con extensión al seno esfenoidal y región prepontina. En dos pacientes se apreció una invasión tumoral 
Tabla 1. Distribución de pacientes según edad, sexo, cuadro clínico, diagnóstico histológico y volumen de la lesión

\begin{tabular}{|c|c|c|c|c|c|}
\hline Casos & Edad & Sexo & Clínica & Histología & $\begin{array}{l}\text { Características imagenológicas y volumen tumoral } \\
\qquad\left(\mathrm{cm}^{3}\right)\end{array}$ \\
\hline Caso 1 & 67 & $\mathrm{~F}$ & Cefalea y diplopia & C. Clásico & $\begin{array}{l}\text { Erosión clival con extensión al seno esfenoidal y región } \\
\text { prepontina, } 30 \mathrm{~cm}^{3}\end{array}$ \\
\hline Caso 2 & 60 & M & Cefalea y diplopia & C. Clásico & $\begin{array}{l}\text { Erosión clival con extensión al seno esfenoidal y región } \\
\text { prepontina, } 57 \mathrm{~cm}^{3}\end{array}$ \\
\hline Caso 3 & 23 & M & $\begin{array}{l}\text { Cefalea, diplopía e } \\
\text { hipoacusia }\end{array}$ & C. Clásico & $\begin{array}{l}\text { Erosión clival con extensión al seno esfenoidal, región } \\
\text { prepontina y ángulo pontocerebeloso, } 125 \mathrm{~cm}^{3}\end{array}$ \\
\hline Caso 4 & 57 & $\mathrm{~F}$ & Cefalea y diplopia & C. Condroide & $\begin{array}{l}\text { Erosión clival con extensión al seno esfenoidal y región } \\
\text { prepontina }, 60 \mathrm{~cm}^{3}\end{array}$ \\
\hline Caso 5 & 65 & M & $\begin{array}{l}\text { Cefalea, diplopía e } \\
\text { hipoacusia }\end{array}$ & C. Condroide & $\begin{array}{l}\text { Erosión clival con extensión al seno esfenoidal, región } \\
\text { prepontina y ángulo pontocerebeloso, } 56 \mathrm{~cm} 3\end{array}$ \\
\hline Caso 6 & 68 & $\mathrm{~F}$ & Cefalea y diplopia & C. Clásico & $\begin{array}{l}\text { Erosión clival con extensión al seno esfenoidal, región } \\
\text { prepontina y seno cavernoso, } 78 \mathrm{~cm}^{3}\end{array}$ \\
\hline Caso 7 & 63 & $\mathrm{~F}$ & Cefalea y diplopia & C. Condroide & $\begin{array}{l}\text { Erosión clival con extensión al seno esfenoidal y región } \\
\text { prepontina, } 90 \mathrm{~cm}^{3}\end{array}$ \\
\hline Caso 8 & 59 & M & Cefalea y diplopia & C. Clásico & $\begin{array}{l}\text { Erosión clival con extensión al seno esfenoidal y región } \\
\text { prepontina, } 34 \mathrm{~cm}^{3}\end{array}$ \\
\hline Caso 9 & 23 & M & Cefalea y diplopia & C. Clásico & $\begin{array}{l}\text { Erosión clival con extensión al seno esfenoidal y región } \\
\text { prepontina, } 10 \mathrm{~cm}^{3}\end{array}$ \\
\hline Caso 10 & 59 & M & Cefalea y diplopia & C. Clásico & $\begin{array}{l}\text { Erosión clival con extensión al seno esfenoidal y región } \\
\text { prepontina, } 55 \mathrm{~cm}^{3}\end{array}$ \\
\hline
\end{tabular}

al ángulo pontocerebeloso y en un caso al seno cavernoso.

Tratamiento (Tabla 2): Todos los pacientes se trataron quirúrgicamente a través de un Abordaje Endonasal Endoscópico Extendido al Clivus (Figura 1), seguidos de radioterapia de intensidad modulada (IMRT) o 3D conformada. Se logró una resección macroscópica total en el $80 \%$, con solo una recidiva tumoral a los 4 años de operados. El $70 \%$ de los pacientes no tuvo complicaciones, se presentaron 2 casos con fístula de líquido cefalorraquídeo, uno de ellos falleció por disfunción de centros nerviosos superiores asociado a meningitis.

En la Figura 2 se realiza un análisis multivariado de variables clínicas, imagenológicas y anatomopatológicas, apreciándose en la dimensión 1 una correlación entre las variables clínicas cefalea y diplopia con la variable imagenológica erosión clival. Se aprecia una relación entre la variable exéresis tumoral y recidiva tumoral, no apreciándose relación de la histología tumoral con el resto de las variables estudiadas.

\section{Discusión}

Uno de los aspectos típicos de los cordomas de la base del cráneo es su crecimiento extradural, generalmente afectando y erosionando el clivus con la ausencia de bordes que delimiten de una forma clara la extensión del tumor. El proceso de tratamiento lleva implícito el tratamiento quirúrgico, el cual siempre es complejo y cuando se realiza por métodos tradicionales se acompaña de una elevada morbimortalidad.

En esta serie la edad promedio fue de 55 años predominando los casos entre la sexta y séptima década de vida lo cual coincide con los estudios de P. Leah ${ }^{1}$ y Abdul-Kareem $\mathrm{Ahmed}^{10}$. En la serie de casos de Raheel Ahmed ${ }^{8}$ y Ke Wang ${ }^{9}$ hubo un franco predominio del sexo masculino lo cual coincide con nuestra serie. La descripción sistemática de la presentación clínica de los cordomas del clivus es escasa, la cual está determinada por el tamaño de la lesión y el eje de crecimiento del mismo, sin embargo, en series de casos reportan cefalea, disturbios visuales, parálisis del VI nervio craneal, defecto motor, dolor cervical, trastornos neuroendocrinos entre otros ${ }^{2}$; nuestros resultados mostraron similitud con estas series siendo la cefalea y la parálisis del VI nervio craneal las que más prevalecieron. Esta sintomatología refleja la invasión a las meninges adyacentes y al canal de Dorello donde transcurre el nervio abductor, respectivamente. En otras series se han encontrado casos asintomáticos un tiempo largo de la enfermedad lo que traduce el crecimiento lento de este tipo de tumor ${ }^{11}$.

En una comparación de la expresión de genes en las diferentes líneas celulares ha revelado una significativa diferencia en la expresión de algunos genes como el MAGEC2 y el SEMA6A los cuales están asociados con una tendencia de los cordomas del clivus a metastizar, proliferar y migrar4; lo que explica porque en algunos casos la evolución clínica es muy tórpida.

Los pacientes con lesiones con volumen tumoral mayores de $70 \mathrm{~cm}^{3}$ tienen alta tasa de recidiva. El índice de recurrencia y los factores de mal pronóstico más consistentes en la mayoría de las series han sido el volumen de la lesión y el grado de resección ${ }^{1,2,5}$. En nuestra serie el volumen tumoral varió entre 10 y $125 \mathrm{~cm}^{3}$ (media 59,5 cm $\mathrm{cm}^{3}$ y el $50 \%$ fue por debajo de $70 \mathrm{~cm}^{3}$. Vellutini ${ }^{3}$ señala en su serie que las exten- 


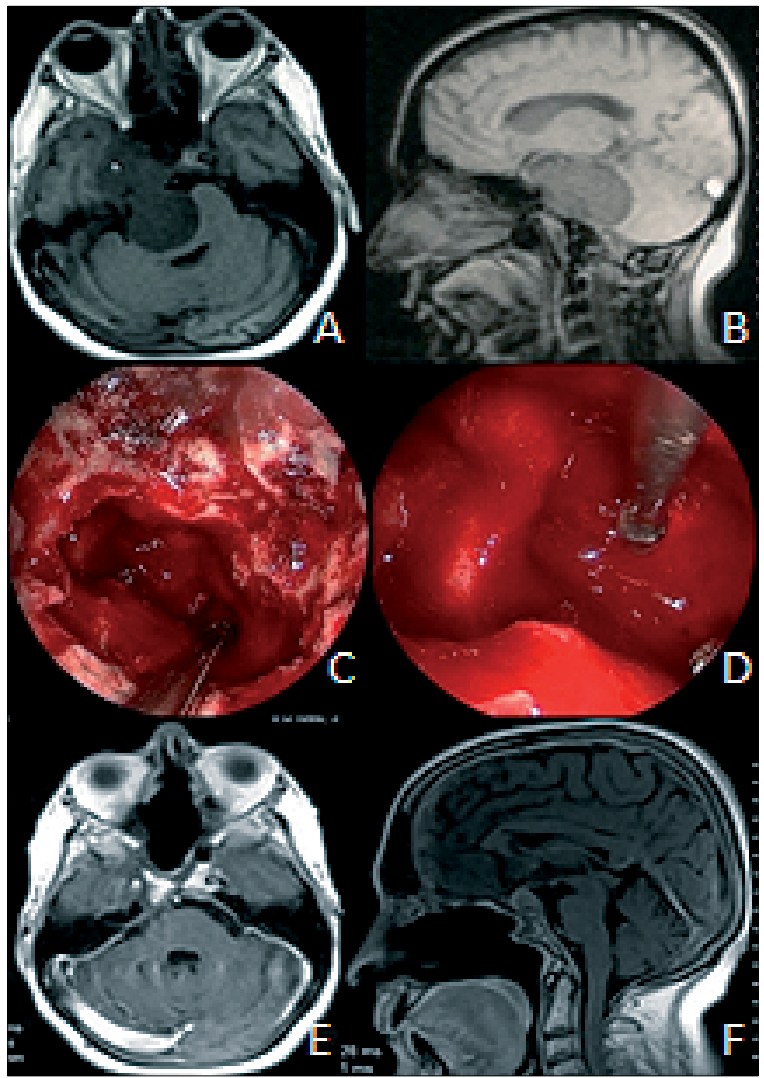

Figura 1. Abordaje Endonasal Endoscópico Extendido al Clivus y Ápex Petroso. A y B. Imágenes preoperatorias, se aprecia lesión tumoral hipointensa en T1 que ocupa la región clival con erosión del clivus e invade el seno cavernoso y región prepontina, con severa compresión del tallo encefálico; C y D. Visualización endoscópica del clivus y seno cavernoso derecho. Se aprecia la carótida paraclival y cavernosa desde una perspectiva anteroinferior; E y F. Imágenes posoperatorias, se evidencia gran resección tumoral con descompresión total del tallo encefálico.

siones laterales de estas lesiones están asociadas también a menos índice de resección total, reportando 48\%. Según el último metaanálisis publicado en 2016 por Labidi y colaboradores $^{12}$ reportan índices de resecciones totales de $39 \%$ y Gui ${ }^{14}$ solo de $24 \%$. En nuestra serie el índice de resección macroscópica total fue superior a la media, siendo de un $80 \%$, lo cual estuvo influenciado por el abordaje endonasal endoscópico empleado que permitió un acceso directo a la lesión, con la mínima retracción de estructuras neurovasculares y con una excelente visualización quirúrgica, así como por la experiencia del equipo quirúrgico. Los cordomas del clivus al originarse en la sincondrosis esfeno-occipital por restos de la notocorda anatómicamente el mayor volumen tumoral se encuentra en línea media, por tanto, el abordaje de inicio seleccionado fue el Endonasal Endoscópico Extendido al Clivus. Cabe destacar que en 2 casos no se pudo lograr una resección macroscópica total, en ambos el tumor tenía una extensión al seno cavernoso o al ángulo pontocerebeloso y no fue posible desde el punto de vista técnico acceder a estos sitios intrincados a pesar de la ampliación del abordaje, estos pacientes presentaron restos tumorales menores de $3 \mathrm{~cm}$ de diámetro mayor, uno de ellos falleció y el otro se mantuvo estable después del tratamiento radiante por 4 años, al cual

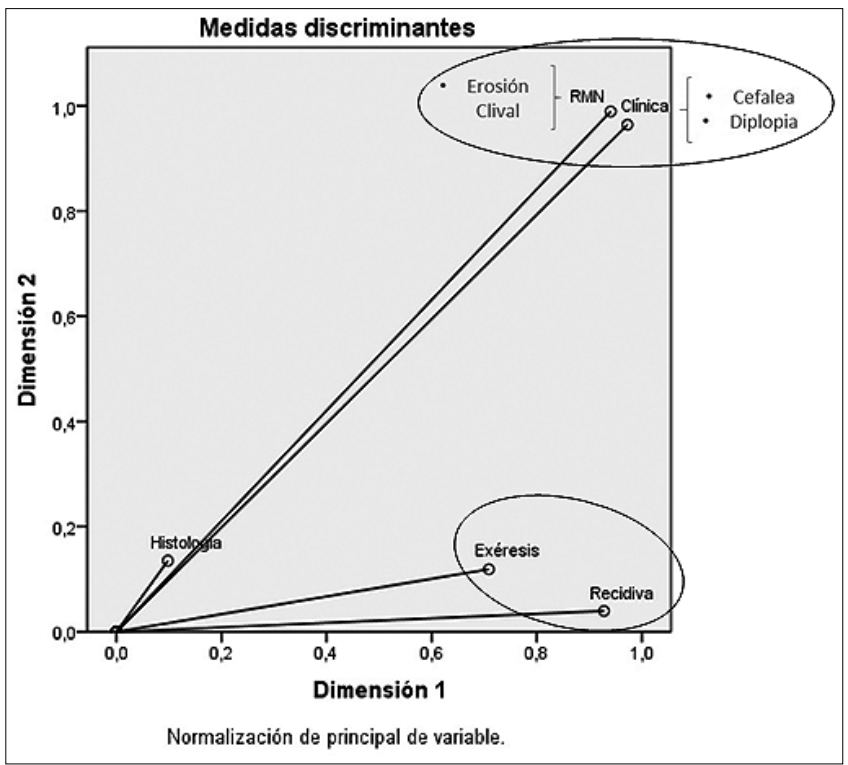

Figura 2. Análisis multivariado de correspondencias múltiples del estatus clínico, localización, exéresis tumoral, índice de recidiva e histología tumoral.

se le realizó un Key Hole Subtemporal y reirradiación.

En el trabajo de Jho y Carrau ${ }^{14}$ se incluyen varias series de tumores del clivus operados por vía endoscópica exclusivamente en los que se reportaron resultados similares a los operados por vía tradicional, pero con menores tasas de complicaciones. Komotar y colaboradores ${ }^{15}$, realizaron una comparación entre el abordaje endoscópico y transcraneal en la que se observó mayor porcentaje de resección total, menos déficit neurológico, menos complicaciones posquirúrgicas y menos recurrencias con la técnica endoscópica. Koutourousiou y colaboradores ${ }^{17}$, señalan que el factor predictor más importante para obtener una resección completa es la experiencia del equipo quirúrgico.

Las complicaciones quirúrgicas fueron pocos frecuentes siendo la fistula del LCR (2 pacientes) la más notables. En uno de los casos existieron complicaciones en cascada que incluyó fístula de LCR, meningitis, disfunción de centros nerviosos superiores y muerte a los 14 días de operado. En ambos casos se colocaron drenajes espinales continuos y reparación directa de la fístula una vez realizado el diagnóstico de la fístula. El colgajo nasoseptal de Hadad modificado, con una base ancha inferolateral desde el arco coanal y colocado en el defecto óseo luego de la grasa y fascia lata fue determinante en los buenos resultados.

En los cordomas del clivus la radioterapia constituye un pilar esencial del proceso de tratamiento. Todos los pacientes recibieron tratamiento coadyuvante con IMRT o 3D conformada, lográndose a los 4 años un $90 \%$ de supervivencia libre de enfermedad.

\section{Conclusiones}

Se evidenció una triada característica en la presentación clínica e imagenológica de los pacientes con cordomas del clivus (tríada de Arbolay-Ortiz): cefalea, diplopía y erosión 


\begin{tabular}{|c|c|c|c|c|c|}
\hline & $\begin{array}{l}\text { Grado de } \\
\text { resección }\end{array}$ & $\begin{array}{l}\text { Radioterapia } \\
\text { (Modalidad) }\end{array}$ & Complicaciones & Recidiva & $\begin{array}{l}\text { Tiempo libre de } \\
\text { enfermedad }\end{array}$ \\
\hline Caso 1 & Total & IMRT & No & No & 7 años \\
\hline Caso 2 & Total & IMRT & Neumoencéfalo & No & 10 años \\
\hline Caso 3 & Subtotal & - & $\begin{array}{l}\text { Fistula del LCR } \\
\text { Meningitis } \\
\text { Fallece a los } 14 \\
\text { días }\end{array}$ & No & - \\
\hline Caso 4 & Total & 3D conformada & No & No & 7 años \\
\hline Caso 5 & Total & IMRT & No & No & 7años \\
\hline Caso 6 & Subtotal & 3D conformada & No & $\mathrm{Si}$ & 4 años \\
\hline Caso 7 & Total & 3D conformada & Fistula del LCR & No & 3años \\
\hline Caso 8 & Total & IMRT & No & No & 5 años \\
\hline Caso 9 & Total & IMRT & No & No & 8 años \\
\hline Caso 10 & Total & IMRT & No & No & 8 años \\
\hline
\end{tabular}

clival, la cual estuvo presente en el $100 \%$ de los pacientes.

El abordaje endonasal endoscópico extendido al clivus realizado por un equipo de experiencia, constituye una opción quirúrgica excelente y prometedora que permite una resección quirúrgica radical en la mayoría los casos con mínimas complicaciones y una supervivencia libre de enfermedad elevada.

\section{Referencias}

1. Leah P, Dower A, Vescovi C, Mulcahy M, Al Khawaja D. Clinical experience of intracranial chordoma - a systematic review and meta-analysis of the literature. J Clin Neurosci. 2018; 53:6-12. PubMed.PMID:29724651.

2. Jahangiri A, Jian B, Miller L, El-Sayed IH, Aghi MK. Skull base chordomas: clinical features, prognostic factors, and therapeutics. Neurosurg Clin N Am. 2013;24(1):79-88.

3. Koutourousiou M, Snyderman $\mathrm{CH}$, Fernández-Miranda J, Gardner PA. Skull base chordomas. Otolaryngol Clin North Am. 2011;44(5):1155-71.

4. Jäger $D$, Lechel $A$, Tharehalli $U$, Seeling $C$, Möller $P$, Barth TFE, et al. U-CH17P, -M and -S, a new cell culture system for tumor diversity and progression in chordoma. Int $\mathrm{J}$ Cancer. 2018;142(7):1369-78. PubMed.PMID:29148152.

5. Culebras D, Torales J, Reyes LA, Zapata L, García S, Roldán P, et al. Cirugía endoscópica endonasal extendida para cordomas y condrosarcomas de clivus: nuestra experiencia en 14 casos. Neurocirugia [Internet]. 2018 [citado 20 Dic 2019];2 9(4):201-8. Disponible en: https://www.sciencedirect.com/science/article/ abs/pii/S1130147318300307.

6. Jahangiri A, Jian B, Miller L, El-Sayed IH, Aghi MK. Skull base chordomas clinical features, prognostic factors, and therapeutics. Neurosurg Clin N Am. 2013;24:79-88.

7. Fujii R, Schlom J, Hodge JW. A potential therapy for chordoma via antibody-dependent cell-mediated cytotoxicity employing NK or high-affinity NK cells in combination with cetuximab. $J$
Neurosurg. 2018;128(5):1419-27. PubMed:PMID:28753113.

8. Ahmed R, Sheybani A, Menezes AH, Buatti JM, Hitchon PW. Disease outcomes for skull base and spinal chordomas: a single center experience. Clin Neurol Neurosurg. 2015;130:67-73. PubMed.PMID:25590662.

9. Wang K, Wang L, Wu Z, Tian K, Ren C, Jia G, et al. Bone invasiveness is associated with prognosis in clivus chordomas. $\mathrm{J}$ Clin Neurosci. 2016;27:147-52. PubMed:PMID:26810468.

10. Ahmed AK, Dawood HY, Arnaout OM, Laws ER, Smith TR. Presentation, treatment, and long-term outcome of intrasellar chordoma: a pooled analysis of institutional, SEER (surveillance epidemiology and end results), and published data. World Neurosurg. 2018;109:e676-83. PubMed:PMID:29061463.

11. Jahangiri A, Jian B, Miller L, El-Sayed IH, Aghi MK. Skull base chordomas: clinical features, prognostic factors, and therapeutics. Neurosurg Clin N Am. 2013;24(1):79-88.

12. Labidi M, Watanabe K, Bouazza S, Bresson D, Bernat AL, George $B$, et al. Clivus chordomas: a systematic review and metaanalysis of contemporary surgical management. J Neurosurg Sci. 2016;60:476-84. PubMed:PMID:27303859.

13. Vellutini Ede AS, Balsalobre L, Hermann DR, Stamm AC. The endoscopic endonasal approach for extradural and intradural clivus lesions. World Neurosurg. 2014;82Suppl 6:S106-15.

14. Gui S, Zong X, Wang X, Li C, Zhao P, Cao L, et al. Classification and surgical approaches for transnasal endoscopic skull base chordoma resection: A 6-year experience with 161 cases. Neurosurg Rev. 2016;39:321-32. PubMed:PMID:26846667.

15. Jho HD, Carrau RL. Endoscopic endonasal transsphenoidal surgery: experience with 50 patients. J Neurosurg.1997;87:44-51.

16. Komotar RJ, Starke RM, Raper DMS, Anand VK, Schwartz TH. The endoscope-assisted ventral approach compared with open microscope-assisted surgery for clival chordomas. World Neurosurg. 2011;76:318-27.

17. Koutourousiou M, Gardner PA, Tormenti MJ, Henry SL, Stefko $S T$, Kassam $A B$, et al. Endoscopic endonasal approach for resection of cranial base chordomas: Outcomes and learning curve. Neurosurgery. 2012;71:614-24. 